\title{
Análise histórica de diagnósticos de enfermagem relacionados a feridas e lesões de pele
}

\section{Historical analysis of nursing diagnoses related to wounds and skin lesions}

\author{
Francisca Graslânia Félix de Sousa' • Vanessa Aguiar Ponte ${ }^{2}$ \\ Maria Girlane Sousa Albuquerque Brandão ${ }^{3}$ \\ Alan Sidney Jacinto da Silva ${ }^{4}$ Lívia Moreira Barros ${ }^{5}$ Thiago Moura Araújo ${ }^{6}$
}

\begin{abstract}
RESUMO
Objetivo:Analisar historicamente mudanças nos diagnósticos de enfermagem relacionados às feridas e lesões de pele. Método: Pesquisa documental, realizada de abril de 2017 a abril de 2019. Fizeram parte da amostra os diagnósticos de enfermagem:Integridade da Pele Prejudicada, risco da Integridade da Pele Prejudicada, Integridade Tissular Prejudicada, Risco da Integridade Tissular Prejudicada, Risco de Lesão por Pressão da Taxonomia II da NANDA de $200 \mathrm{I}$ a 2018. Resultado:A principal mudança foi nas características definidoras de Integridade da pele prejudicada.A definição de Risco da integridade da pele prejudicada passou por quatro mudanças. O diagnóstico Integridade tissular prejudicada modificou a definição e fatores relacionados. Houve alteração no diagnóstico Risco de Úlcera por pressão para Risco de lesão por pressão. Conclusão:As mudanças em terminologias e acréscimo de informações nos diagnósticos foram realizadas no intuito de favorecer a sistematização da assistência de enfermagem e fomentar a melhor identificação dos diagnósticos.
\end{abstract}

Palavras-Chave: Ferimentos e lesões; Diagnóstico de Enfermagem;Assistência de Enfermagem.

\begin{abstract}
Objective: To analyze historically changes in nursing diagnoses related to wounds and skin lesions. Method: Documentary research, conducted from April 2017 to April 2019. The sample included the nursing diagnoses: Harmed Skin Integrity, Harmed Skin Integrity Risk, Harmed Tissue Integrity, Harmed Tissue Integrity Risk, Risk of Injury by NANDA Taxonomy II Pressure from 200I to 2018. Result: The main change was in the defining characteristics of impaired skin integrity. The definition of Risk of impaired skin integrity has undergone four changes. The diagnosis Impaired tissue integrity changed the definition and related factors. There was a change in the diagnosis Pressure Ulcer Risk to Risk of Pressure Injury. Conclusion:The changes in terminology and the addition of information in diagnoses were made in order to favor the systematization of nursing care and foster better identification of diagnoses.

Keywords: Wounds and injuries; Nursing Diagnosis; Nursing care.
\end{abstract}




\section{INTRODUÇÃO}

A pele é o maior órgão do corpo humano e representa aproximadamente $16 \%$ do peso corporal total. É a interface primária entre os ambientes interno e externo, que atua como barreira para proteger o corpo de uma série de estressores ambientais e manter a homeostase, para evitar a perda de água e eletrólitos não regulamentada.'

Mesmo sendo um grande órgão, incontáveis fatores pode prejudicar a integridade da pele, o que resulta em descontinuidade dos tecidos e desenvolvimento de feridas. Fatores como pressão, traumas mecânicos, químicos, físicos e isquêmicos e a intenção no caso de cirurgias, podem resultar em variados tipos de lesões de pele, que constituem sério problema de saúde pública e de abrangência mundial em razão do número de pessoas com alterações na integridade da pele. No Brasil, aproximadamente $3 \%$ da população do país têm algum tipo de lesão de pele. ${ }^{2}$

Indivíduos que apresentam lesões de pele necessitam de assistência de enfermagem para um tratamento adequado, além da prevenção de agravos. Esse cuidado está pautado na Sistematização da Assistência de Enfermagem (SAE), que se apresenta como uma forma de organizar a assistência de enfermagem, de modo a intervir de acordo com as reais necessidades do paciente e promover sua rápida recuperação. ${ }^{3}$

A utilização da SAE ou do Processo de Enfermagem (PE) é um dos principais recursos que possibilita ao enfermeiro utilizar suas competências técnicas e científicas, como também aplicar o cuidado humanizado na assistência ao paciente. ${ }^{4}$

A enfermagem possui vários sistemas de classificação para - desenvolvimento das etapas do PE, e entre eles destacase a North American Nursing Diagnosis Association International (NANDA-I), por ser a mais reconhecida e difundida no mundo e no Brasil.Utiliza raciocínio e julgamento clínico do enfermeiro para diagnosticar as respostas humanas a problemas de saúde e processos de vida reais ou potenciais, e possibilita a utilização de linguagem padronizada para melhor comunicar os fenômenos de interesse da prática da enfermagem, a documentação e avaliação do cuidado. ${ }^{5}$

Os diagnósticos de enfermagem (DE) relacionados às feridas e lesões de pele merecem destaque por se tratar de uma etapa dinâmica, sistemática, organizada e complexa do processo de enfermagem, significando não apenas simples listagem de problemas, mas uma fase que envolve avaliação crítica e tomada de decisão. ${ }^{6}$

Os diagnósticos relacionados com o cuidado a pele e ferimentos são recorrentes em estudos da temática. Pesquisas em hospitais do Nordeste e Sudeste brasileiro identificaram respectivamente, que $100 \%$ dos pacientes possuíam diagnóstico de Integridade tissular prejudicada, e $60 \%$, Integridade da pele prejudicada. ${ }^{7,8}$

A utilização dos diagnósticos de enfermagem no cuidado ao paciente com feridas é importante contribuição para uma avaliação contínua do tratamento instituído, pois esta etapa do PE irá direcionar a prática da assistência, permitindo acompanhar a evolução da ferida e a resposta do paciente frente ao tratamento. ${ }^{9}$

Os diagnósticos de enfermagem no campo das lesões de pele e feridas cutâneas são imprescindíveis para uma prática assistencial organizada, uma vez que, por eles serão planejados e realizados os cuidados de enfermagem necessários para determinar a melhora da lesão, e, consequentemente o restabelecimento da saúde do cliente.

O estudo tem por objetivo analisar historicamente as mudanças nos diagnósticos de enfermagem relacionados às lesões de pele e feridas.

\section{MÉTODO}

\section{Tipo de pesquisa}

Estudo qualitativo, do tipo documental. $O$ corpus documental foi composto por edições dos livros Diagnósticos de enfermagem da NANDA-I.

\section{População e Amostra}

A população do corpus documental foram oito edições dos Diagnósticos de enfermagem da NANDA-I, referente aos anos de $200 \mathrm{I}$ a 2018. Constituíram a amostra cinco diagnósticos de enfermagem relacionados diretamente com feridas e lesões de pele: Integridade da pele prejudicada, risco da integridade da pele prejudicada, integridade tissular prejudicada, risco da integridade tissular prejudicada, risco de lesão por pressão. Estes diagnósticos pertencem ao domínio II: segurança/ proteção; classe 2: lesão física.

\section{Coleta de Dados}

A busca dos documentos ocorreu no período de abril de 2017 a setembro de 2019 . A extração dos dados foi realizada por meio de instrumento no Microsoft Word $\AA$ criado pelos autores, contendo: ano de publicação, ano, taxonomia II, definição, características definidoras e fatores relacionados.

\section{Critérios de inclusão}

Foram incluídas as versões da NANDA-I disponíveis nas bibliotecas da Universidade da Integração Internacional da Lusofonia Afro-Brasileira e Escola de Enfermagem de Ribeirão Preto da Universidade de São Paulo e/ou com versão online em e-book, que apresentassem diagnósticos de enfermagem relacionados com feridas e lesões de pele. Foram excluídas versões inacessíveis nas referidas bibliotecas universitárias e que também não estavam disponíveis em e-book, sendo excluídas, portanto, três versões.

\section{Organização dos dados}

Os resultados foram organizados em quadros, com apresentação de título, definição, características definidoras, fatores relacionados e os anos das respectivas publicações. Para os diagnósticos de risco, apresentam-se título, definição, ano e fatores de risco.

Considerou-se para análise dos resultados a leitura dos diagnósticos, além de busca na literatura de contextos históricos que justificassem determinadas mudanças.

\section{RESULTADOS}

$\mathrm{Na}$ pesquisa, analisou-se cronologicamente as mudanças ocorridas nos diagnósticos de enfermagem relacionados a feridas e lesões de pele entre os anos de 2001 à 2018 , tendo em vista a prevalência desses diagnósticos em vários estudos que identificam as características definidoras e fatores de riscos presentes nos indivíduos.

O diagnóstico de enfermagem Integridade da Pele Prejudicada não passou por mudanças com relação ao título da taxonomia II e quanto a sua definição durante o período em análise.

$\mathrm{Na}$ análise das características definidoras, observa-se que: 
"invasão de estruturas do corpo; destruição da camada da pele (derme); rompimento da superfície da pele (epiderme)" foram mantidas até as versão 20I2-20I4. Na versão seguinte (2015-2017) apresenta: "alteração na integridade da pele e matéria estranha perfurando a pele”. Na última versão (20182020) foram acrescidos os itens "Área localizada quente ao toque; dor aguda; hematoma; sangramento e vermelhidão" nas características definidoras.

Nos fatores relacionados externos do diagnóstico de enfermagem Integridade da Pele Prejudicada, destaca-se que até a versão 2005-2006 o item "hipertermia ou hipotermia" eram associados em um único fator, já na versão seguinte (2007-2008) foram separados.

Nos fatores relacionados internos, observa-se a troca de estado metabólico alterado por alteração metabólica na versão (2005-2006) e por estado metabólico prejudicado em 20122014, déficit imunológico até a versão (2007-2008), deficiência imunológica na versão (20I2-20I4) por imunodeficiência em 20I5-20I7; estado nutricional alterado desequilibrado por nutrição inadequada"; fatores de desenvolvimento por mudança hormonal; "mudança na pigmentação por alteração na pigmentação"; "mudança no estado hídrico por alteração no volume de líquidos”; "mudanças no turgor por alterações no turgor da pele"; "proeminências ósseas por pressão sobre saliência óssea"; sensação prejudicada por alterações na sensibilidade." com destaque para a inclusão de agente químico lesivo e seus exemplos, embora já tenha sido contemplado o fator substância químicas nas edições anteriores.

Percebe-se que a definição "Estar em risco de pele ser alterada de forma adversa" manteve-se nos anos de 200I a 2006 (três edições) e alterou de "estar em risco de pele...." para "risco de pele...", que manteve-se de 2007 à 201 I (duas edições). $\mathrm{Na}$ edição de 20|2-20।4 manteve somente a palavra "risco", modificando a definição para "Risco de epiderme e/ ou derme alterada".

Quadro I.Descrição histórica do Diagnóstico de Enfermagem Integridade da Pele prejudicada (200I-20I8) da Taxonomia II da NANDA. Redenção/CE - Brasil, 2019.

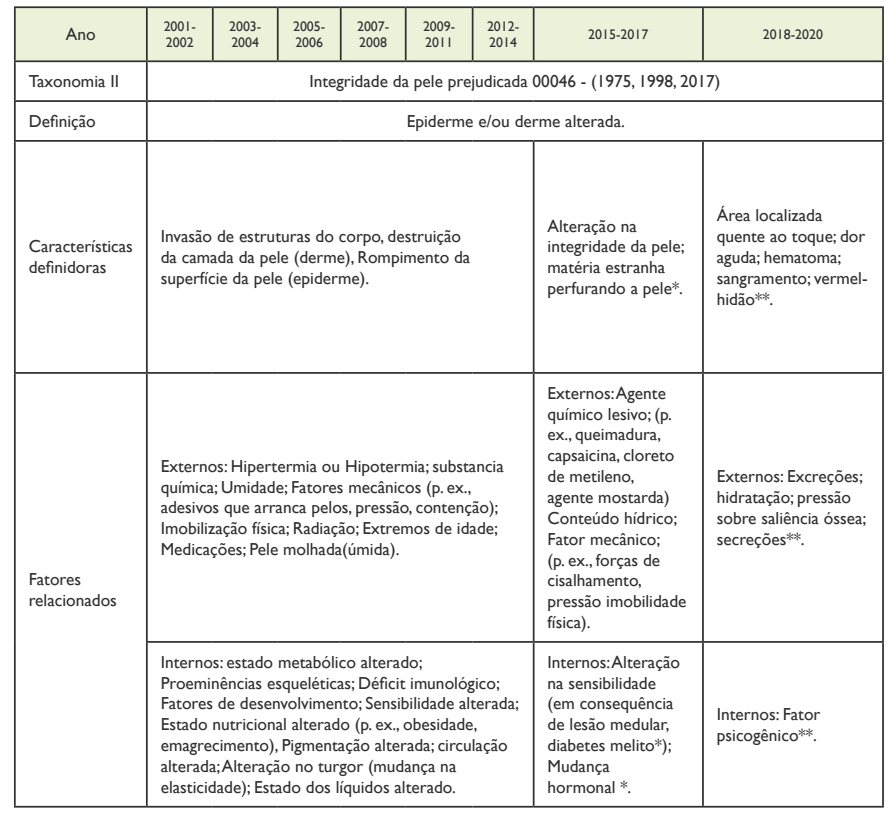

*Alteração na terminologia; **Acréscimo de itens. Fonte: NANDA (200I-2018)
Aponta-se a permanência dos mesmos fatores de risco relacionados ao DE "Risco de Integridade da Pele Prejudicada" até a versão (20I2-20I4), publicada no Brasil. Na versão 20I52017 os fatores externos e internos passaram por acréscimos com destaque para a inclusão de agente químico lesivos e seus exemplos (Quadro I). Na útima versão (20I8-2020) também houve acréscimos nos fatores externos e internos, sendo respectivamente: excreções; hidratação; pressão sobre saliência óssea; secreções, e fator psicogênico.

Destaca-se que até a versão (2005-2006) apresentava-se o fator de risco "pele úmida", que passoua apresentar "pele molhada”, e na versão (20I5-20l7), hidratação. E a alteração de radiação por radioterapia.

No quadro 2, percebe-se que nos fatores de risco internos, que "estado nutricional desequilibrado foi alterado para nutrição inadequada", "fatores imunológicos por imunodeficiência", "medicamentos por agentes farmacológicos", "mudança na pigmentação por alteração no pigmento da pele", "estado metabolico prejudicado por alteração no metabolismo", "proeminencias ósseas por pressão sobre saliências ósseas", “sensações prejudicadas por alteração na sensibilidade”.

Quadro 2. Descrição histórica do Diagnóstico de Enfermagem Risco de Integridade da Pele prejudicada (200I-20I8) da Taxonomia II da NANDA. Redenção/CE - Brasil, 2019.

\begin{tabular}{|c|c|c|c|c|c|c|c|c|}
\hline Ano & $\begin{array}{l}2001 . \\
2002\end{array}$ & \begin{tabular}{|l}
$2003 \cdot$ \\
2004
\end{tabular} & $\begin{array}{ll}2005 . \\
2006\end{array}$ & $\begin{array}{ll}2007- \\
2008\end{array}$ & $\begin{array}{l}2009 . \\
2011 .\end{array}$ & $2012-2014$ & 2015-2017 & $2018-2020$ \\
\hline $\begin{array}{c}\text { Taxonomia } \\
\|\end{array}$ & \multicolumn{8}{|c|}{ Risco da integridade da pele prejudicada- $00047(1998,2010,2013,2017)$} \\
\hline Definição & \multicolumn{3}{|c|}{$\begin{array}{l}\text { Estar em risco de } \\
\text { pele ser alterada de } \\
\text { forma adversa }\end{array}$} & \multicolumn{2}{|c|}{$\begin{array}{l}\text { Risco de pele } \\
\text { ser alterada } \\
\text { de forma } \\
\text { adversa.* }\end{array}$} & $\begin{array}{l}\text { Risco de } \\
\text { epiderme el } \\
\text { ou derme } \\
\text { alterada. * }\end{array}$ & $\begin{array}{l}\text { Vulnerabilidade } \\
\text { na alteraçáa } \\
\text { da pele elou } \\
\text { derme, que pode } \\
\text { comprometer a } \\
\text { saúde. }\end{array}$ & $\begin{array}{l}\text { Suscetibilidade } \\
\text { a alteração na } \\
\text { epiderme e/ou } \\
\text { derme que pode } \\
\text { comprometer a } \\
\text { saúde.* }\end{array}$ \\
\hline \multirow[t]{2}{*}{$\begin{array}{l}\text { Fatores } \\
\text { de risco }\end{array}$} & \multicolumn{6}{|c|}{$\begin{array}{l}\text { Externos: Radiação; imobilização física; Fatores mecânicos } \\
\text { (p. ex adesivos que arranca pelos, pressão, contenção) } \\
\text { hipertermia; hipotermia; umidade; substância química; } \\
\text { excreçōes e/ou secreçôes; pele molhada (úmida), idades } \\
\text { extremas. }\end{array}$} & $\begin{array}{l}\text { Externos: } \\
\text { Agente químico } \\
\text { lesive (p. ex., } \\
\text { queimadura, cap- } \\
\text { saicina, cloreto } \\
\text { de metileno, } \\
\text { agente mostarda) } \\
\text { *; hidrataçäo** }\end{array}$ & $\begin{array}{l}\text { Externos: Excrę̧ōes; } \\
\text { hidrataçăo; pressão } \\
\text { sobre saliencia óssea; } \\
\text { secreçôes*\%. }\end{array}$ \\
\hline & \multicolumn{6}{|c|}{$\begin{array}{l}\text { Internos: Medicação; proeminência esquelética; fatores } \\
\text { imunológicos; fatores de desenvolvimento; sensibilidade } \\
\text { alterada; estado metabólico alterado; circulação alterada; } \\
\text { alteraçōes no turgor da pele (mudança na elasticidade); } \\
\text { alteração no estado nutricional (p. ex., obesidade, emagrec- } \\
\text { imento); psicogênicos. }\end{array}$} & $\begin{array}{l}\text { Internos: } \\
\text { mudança } \\
\text { hormonal* }\end{array}$ & $\begin{array}{l}\text { Internos: Fator } \\
\text { psicogênico**. }\end{array}$ \\
\hline
\end{tabular}

*Alteração na terminologia;**Acréscimo de itens. Fonte: NANDA (200I-20I8).

$\mathrm{Na}$ análise do DE Integridade Tissular Prejudicada, observou-se que, a definição manteve-se nas edições de $200 \mathrm{I}$ à 2014 (seis edições), excluindo a "pele" e acrescentando "tecido tegumentar, fáscia muscular, músculo, tendão, osso, cartilagem, capsula articular e/ou ligamento" na versão (20 I52017) e manteve-se na última edição (Quadro 3). 
Quadro 3. Descrição histórica do Diagnóstico de Enfermagem Integridade Tissular prejudicada (200I-20I8) da Taxonomia II da NANDA. Redenção/CE - Brasil, 2019.

\begin{tabular}{|c|c|c|c|c|c|c|c|}
\hline Ano & $\begin{array}{l}2001- \\
2002\end{array}$ & $\begin{array}{l}2003- \\
2004\end{array}$ & $\begin{array}{l}2005- \\
2006\end{array}$ & $\begin{array}{l}2007 . \\
2008\end{array}$ & \begin{tabular}{l|l}
$2009-$ & $2012-$ \\
2011 & 2014
\end{tabular} & $2015-2017$ & $2018-2020$ \\
\hline Taxonomia II & \multicolumn{7}{|c|}{ Integridade tissular prejudicada-00044 $(1986,2013,2017)$} \\
\hline Definição & \multicolumn{5}{|c|}{$\begin{array}{l}\text { Dano a membranas mucosas, córnea, pele, ou } \\
\text { tecidos subcutâneos. }\end{array}$} & $\begin{array}{l}\text { Alteração na } \\
\text { integridade da pele; } \\
\text { matéria estranha } \\
\text { perfurando a pele*. }\end{array}$ & $\begin{array}{l}\text { Área localizada } \\
\text { quente ao toque; dor } \\
\text { aguda; hematoma; } \\
\text { sangramento; vermel- } \\
\text { hidão**. }\end{array}$ \\
\hline $\begin{array}{l}\text { Características } \\
\text { definidoras }\end{array}$ & \multicolumn{5}{|c|}{$\begin{array}{l}\text { Tecido destruído, tecido lesado (p. ex., córnea, } \\
\text { mucosas, pele ou tecido subcutâneo. }\end{array}$} & $\begin{array}{l}\text { Tecido destruído, } \\
\text { tecido lesado.* }\end{array}$ & $\begin{array}{l}\text { Área localizada } \\
\text { quente ao toque; } \\
\text { dano tecidual; dor } \\
\text { aguda; hematoma; } \\
\text { sangramento; } \\
\text { vermelhidado.** }\end{array}$ \\
\hline $\begin{array}{l}\text { Fatores } \\
\text { relacionados }\end{array}$ & \multicolumn{5}{|c|}{ 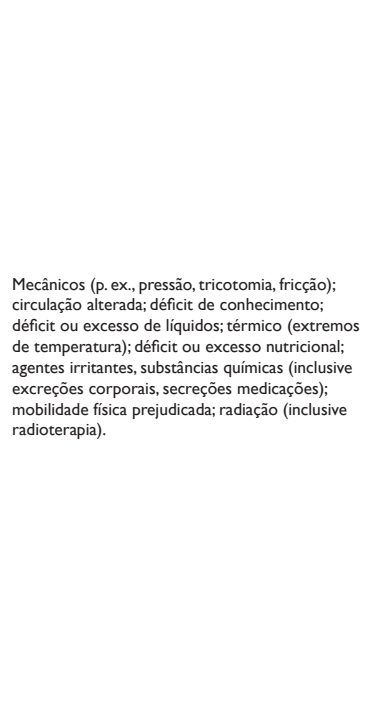 } & 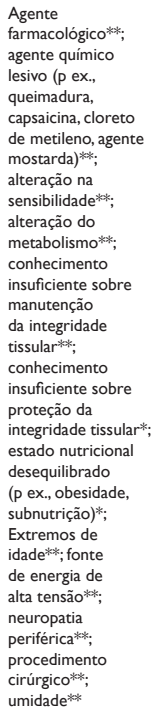 & $\begin{array}{l}\text { Volume de líquidos } \\
\text { deficiente; volume de } \\
\text { líquidos excessivo.* }\end{array}$ \\
\hline
\end{tabular}

*Alteração na terminologia; **Acréscimo de itens. Fonte: NANDA (2001-2018)

Os quadros ( 1 a 3 ) apresentam pequenas mudanças de terminologias que não foram consideradas na construção das tabelas como, por exemplo: medicação por agente farmacológico, pois foram consideradas na pesquisa mudanças não semânticas.

Observa-se a troca de "circulação alterada por circulação prejudicada"; "déficit de conhecimento por conhecimento insuficiente..."; "excesso de líquidos por volume de líquidos excessivo"; "extremos de temperatura por extremo de temperatura ambiental"; "fatores nutricionais por estado nutricional desequilibrado"; "radiação por radioterapia”.

Atualmente a preocupação por descrever DE relacionados à pele emerge em trazer mais $\mathrm{DE}$ relacionado com esse agravo. Nessa lógica a versão da NANDA 20I5-2017 apresentou dois novos DE relacionados às feridas: Risco de Integridade tissular prejudicada (00248) e Risco de úlcera por pressão (00249). Contudo, na última versão (20|8-2020), houve alteração do diagnóstico Risco de úlcera por pressão (00249) para Risco de lesão por pressão (00249) (Quadro 4).
Quadro 4. Descrição dos Novos Diagnóstico de Enfermagem relacionados a feridas e lesões de pele (2015-20I7/ 20I8-2020) da Taxonomia II da NANDA. Redenção/CE - Brasil, 2019.

\begin{tabular}{|c|c|}
\hline \multicolumn{2}{|c|}{ Novos diagnósticos de enfermagem da taxonomia II da NANDA 2015-2017 } \\
\hline Diagnóstico/Definição & Fatores de risco \\
\hline $\begin{array}{l}\text { Risco de Integridade tissular prejudicada (00248) } \\
\text { Vulnerabilidade a dano em membrana mucosa, córnea, } \\
\text { sistema tegumentar, fáscia muscular, músculo, tendão, } \\
\text { osso, cartilagem, cápsula articular e/ou ligamento, que } \\
\text { pode comprometer a saúde. }\end{array}$ & $\begin{array}{l}\text { Externos: agente químico lesivo (p ex., queimadura, cap- } \\
\text { saicina, cloreto de metileno, agente mostarda), alteraçăo } \\
\text { na sensibilidade, alteraçăa o metabolismo, circulaçăo } \\
\text { prejudicada, conhecimento insuficiente sobre manutenção } \\
\text { da integridade tissular, conhecimento insuficiente sobre } \\
\text { proteção da integridade tissular, estado nutricional } \\
\text { desequilibrado (p ex., obesidade, subnutrição), Extremos } \\
\text { de idade, extremos de temperatura ambiental, fator } \\
\text { mecânico, fonte de energia de alta tensão, mobilidade } \\
\text { prejudicada, neuropatia periférica, procedimento cirúr- } \\
\text { gico, terapia por radiação, umidade, volume de liquido } \\
\text { insuficiente, volume excessivo de liquidos. }\end{array}$ \\
\hline $\begin{array}{l}\text { Risco de úlcera por pressão(00249) } \\
\text { Vulnerabilidade a lesão localizada na pele e/ou tecido } \\
\text { subjacente, normalmente sobre saliência óssea, em } \\
\text { consequência de pressão, ou pressão combinada com } \\
\text { forças de cisalhamento. }\end{array}$ & $\begin{array}{l}\text { ADULTO: escore na Escala de Braden< } 18 \text {, CRIANÇA: } \\
\text { Escala de Braden } Q<16 \text {, agentes farmacológicos (p. } \\
\text { ex., anestesia geral, vasopressores, antidepressivos, } \\
\text { norepinefrina), alteração na função cognitiva, alteração na } \\
\text { sensibilidade, anemia, atrito em superficie, baixo escore } \\
\text { na escala de Riskk, , rirculação prejudicada, conhecimento } \\
\text { insuficiente do cuidador sobre prevenção de ulcera } \\
\text { por pressão. }\end{array}$ \\
\hline \multicolumn{2}{|c|}{ Novo diagnóstico de enfermagem da taxonomia II da NANDA 20|8-2020 } \\
\hline $\begin{array}{l}\text { Diagnóstico/Definição } \\
\text { Definição }\end{array}$ & Fatores de risco \\
\hline $\begin{array}{l}\text { Risco de Lesão por pressão (00249) } \\
\text { Suscetibilidade a lesão localizada da pele e/ou tecido } \\
\text { subjacente, normalmente sobre saliência óssea, em conse- } \\
\text { quênncia de pressão, ou pressãa combinada com forças de } \\
\text { cisalhamento (NPUAP, 2007). }\end{array}$ & 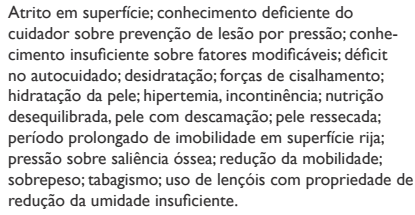 \\
\hline
\end{tabular}

Fonte: NANDA (2015-2018)

O diagnóstico Risco de Lesão por Pressão, foi aprovado em 2013 e revisado em 2017 , com nível de evidência 2.2 .

\section{DISCUSSÃO}

A NANDA apresenta cinco DE relacionados as feridas e lesões de pele. Dentres esses, os DE Integridade da pele prejudicada e Integridade da pele prejudicada classificamse como DE com foco no problema real. Os DE Risco da integridade da pele prejudicada, Risco de lesão por pressão e Risco da integridade tissular prejudicada classificam - se em DE de risco.

A pele consiste na barreira protetora do organismo contra agentes internos e externos. No entanto, inúmeras alterações podem acometer a integridade desta estrutura. A enfermagem deve, portanto, realizar uma assistência pautada na prevenção, avaliação e tratamento dessas lesões, pautada nos fatores de risco, características definidoras e fatores relacionados. Este conhecimento é fundamental para a identificação de um DE e a indicação de tecnologias adequadas para a prevenção e o tratamento de lesões e/ou feridas. ${ }^{10}$

Os diagnósticos de enfermagem são destaques em estudos relacionados à $\mathrm{DE}$ de feridas e lesões de pele, geralmente em ambientes hospitalares e em setores críticos, como Unidades de Terapia Intensiva (UTI) adulta e pediátrica."

Dentre os principais diagnósticos de enfermagem para pacientes em unidades de saúde hospitalares está o DE Integridade da pele prejudicada. ${ }^{2}$ Pesquisa em hospital do Nordeste brasileiro identificaram que o DE Integridade da pele prejudicada está entre os mais prevalentes." A compreensão da importância dos diagnósticos e da prevenção é primordial para que se evitem lesões. ${ }^{13}$

No DE Integridade da Pele Prejudicada a modificação mais significativa foi na definição, em 2015 foi definido como "Vulnerabilidade na alteração da pele e/ou derme, que pode comprometer a saúde”. Na edição 2018, mudou-se o termo 
"Vulnerabilidade" por "Suscetibilidade". Dessa maneira, a vulnerabilidade/suscetibilidade antecede o risco e, ao buscar - entendimento das mediações presentes no processo saúde-doença, promove possibilidades distintas para o seu enfrentamento. ${ }^{14}$

Historicamente, o DE Integridade da Pele Prejudicada é apontado como o mais relevante relacionado à pele e passou por mudanças significativas na versão (20I5-20l7) da NANDA. Ressalta-se que as alterações não tiveram impacto significativo na definição, e sim, nas características definidoras e fatores relacionados. Isso revela que os enfermeiros têm se preocupado em identificar sinais e sintomas e fatores etiológicos necessários para compor o raciocínio crítico na avaliação de lesões de pele e feridas.

O DE Risco da Integridade da Pele Prejudicada presente na taxonomia NANDA é um DE que pode nortear os cuidados preventivos para clientes que estejam em risco de desenvolver lesões de pele. Pesquisa realizada em hospital do Sudeste brasileiro identificou que o diagnóstico de enfermagem mais prevalente foi Risco da Integridade da Pele Prejudicada (35\%). ${ }^{15}$

Em casos que há diagnóstico inicial de risco de integridade de pele prejudicada, deve-se ofertar uma assistência preventiva ao cliente, uma que vez o risco poderá evoluir para inúmeras situações de respostas humanas, como infecção, dor, lesão de pele e interferir negativamente na qualidade da assistência e no quadro clínico do paciente.

Atualmente as medidas mais indicadas como forma de prevenção do risco de lesão é a hidratação da pele, roupa de cama esticada, massagem, orientação, cobertura profilática, aplicação da escala de Braden e proteção de proeminências ósseas.

ODE IntegridadeTissular Prejudicada se manteve estável até a versão (20I2-20l4), contudo, na transição para (20I5-20l7) houve mudança na definição, que passou a ter um sentido mais amplo. Estudos na Europa e África identificaram que lesões de pele são problemas recorrentes em unidade de atendimento à população infantil, mais de $96 \%$ das crianças internadas tinham diagnóstico de integridade tissular prejudicada. ${ }^{16,17}$

A natureza das alterações no diagnóstico permite inferir com base em dados empíricos que se faz necessário para aprimorar o cuidado aos indivíduos. $\mathrm{Na}$ definição, observa-se que na edição (20I5-20I7) foram detalhadas as estruturas que podem ser atingidas com a integridade tissular prejudicada no indivíduo.

$\mathrm{Na}$ estrutura taxonômica da NANDA- I, referente ao domínio I I e classe 2, consta somente um DE para a situação de risco de dano à pele, denominado Risco de integridade da pele prejudicada, definido como "risco de epiderme e/ ou derme alterados". Pela sua definição se vê que é um DE que pode ser usado para diversas situações de risco de alteração na pele.

$\mathrm{Na}$ versão (2018-2020) da NANDA, houve alteração do diagnóstico Risco de úlcera por pressão para Risco de lesão por pressão. Atualmente, as taxas globais de prevalência de lesão por pressão (LPP) em hospitais giram em torno de $12 \%$ em unidades de cuidados gerais e de $23 \%$ em unidades de cuidados agudos. Esses índices elevados apontam para um problema que merece investigação, uma vez que, na maioria das vezes, a LPP pode ser evitável, pela identificação dos fatores de risco e pela implementação de medidas preventivas. Assim, o enfermeiro necessita coletar informações relacionadas ao risco de LPP e, de acordo com seu julgamento clínico, estabelecer um diagnóstico de enfermagem (DE) acurado, de forma a embasar o cuidado a quem for vulnerável. ${ }^{18}$

Assim, inserir os diagnósticos de enfermagem no cuidado aos pacientes com feridas é fundamental para uma avaliação contínua do tratamento instituído, pois esta etapa do PE irá direcionar a prática da assistência, permitindo acompanhar a evolução da ferida e a resposta do paciente frente ao tratamento. ${ }^{19}$

Destaca-se como limitação a ausência de todas as edições da NANDA - I, mediante a dificuldade de acesso as versões mais remotas.

\section{CONCLUSÃO}

A realização desta análise histórica possibilitou identificar as principais mudanças ocorridas nos DE relacionados a feridas e lesões de pele da Taxonomia II da NANDA, desde 200I até a versão mais atual (20l8).

A principal mudança no DE Integridade da pele prejudicada foi nas características definidoras, enquanto no DE Risco da integridade da pele prejudicada houve alteração na definição. No DE Integridade tissular prejudicada também houve alteração na definição e acréscimos nos fatores relacionados.

Houve inserção dos novos DE relacionados a lesões de pele, na NANDA, que se justificou pela necessidade encontrada na literatura.

Salienta-se a relevância a realização de novos estudos que apresentem resultados cada vez mais promissores sobre os diagnósticos de enfermagem relacionados às feridas e lesões de pele.

\section{REFERÊNCIAS}

1. Roger M, Fullard N, Costello L, Bradbury S, Markiewicz $\mathrm{E}$, O'Reilly S, et al. Bioengineering the microanatomy of human skin. J. Anat. 2019; 234(4): 438-455.

2. Oliveira FP, Oliveira BGRB, Santana RF, Silva BP, Candida JSC. Nursing interventions and outcomes classifications in patients with wounds: cross-mapping. Rev Gaúcha Enferm. 2016; 37(2):e55033.

3. Carvalho IM, Ferreira DKS, Nelson ARC, Duarte FHS, Prado NCC, Silva RAR. Systematization of nursing care in mediate post-operative of cardiac surgery. Rev Fund Care Online. 2016; 8(4):5062-5067.

4. Júnior SLAM, Santos ASL, Ribeiro BP, Oliveira F, Freitas MG; Ferreira MZJ. Diagnósticos de Enfermagem à Criança com Queimadura no Pronto-Socorro Infantil: uma revisão integrativa. Rev Enfermagem Atual InDerme. 2019; 84 (22): 97-I0I.

5. North American Nursing Diagnosis Association (NANDA). Diagnósticos de enfermagem da NANDA: definições e classificação - 2009/20I I. Regina Machado Garcez, tradutora. Porto Alegre (RS):Artmed; 2010.

6. Araújo TM, Araújo MFM, Caetano JÁ, Galvão MTG, Damasceno MMC. Diagnósticos de enfermagem para pacientes em risco de desenvolver úlcera por pressão. Rev Bras Enferm. 201 I; 64(4): 67I-6.

7. Silva MR, Silva DO, Santos EC, Oliveira PP, Sales AS, Rodrigues $A B$. Diagnósticos, resultados e intervenções de enfermagem para pessoas submetidas a cirurgias ortopédicas e traumatológicas. Rev enferm UFPE on line. 20 I 7; I I (Supl. 5):2033-45.

8. Grassi MF, Dell'Acqua MC, Jensen R, Fontes CM, Guimarães HC.Diagnósticos, resultados e intervenções 
de enfermagem em pacientes com lesão renal aguda. Acta Paul Enferm. 20I7; 30(5):538-45.

9. Oliveira IC, Veríssimo RCSS, Bastos MLA, ngrid Martins Leite Lúcio. A frequência dos diagnósticos de enfermagem em pacientes com feridas. Rev enferm UFPE on line. 2014; 8 (7): 1937-46.

10. Favreto FJL, Betiolli SE, Silva FB, Campa A. O papel do enfermeiro na prevenção, avaliação e tratamento das lesões por pressão. Rev Gestão \& Saúde. 2017; I7(2):3747.

11. Guedes DMB, Rossato LM, Oliveira EA. Diagnósticos de enfermagem mais frequentes em uma unidade de terapia intensiva pediátrica. Rev Enferm UFSM. 20I 5;5(3):476-485.

12. Ribeiro KRA, Gonçalves FAF, Borges MM, Loreto RGO; Amaral MS.Postoperative Myocardial Revascularization: Possible Diagnosis And Nursing Interventions. Rev Fund Care Online. 2019; I I(3):80 I-808.

13. Teófilo FKS, Silva AVSE, Lima KJ, Dantas APF, Silva VA. Lesões de pele em recém-nascido: revisão integrativa. Rev Enfermagem Atual In Derme. 2019; 86(26): I - I7.

14. Sousa FGM, Erdmann AL. Vulnerabilidade: Análise de conceito para apoiar as práticas de cuidado em saúde. Invest Qualitativa em Saúde. 20 I5; I (I): 559-562.

15. Marcon C, Vicari G, Poltronieri P, Maffissoni A, Caregnatto KDA, Argenta C, Adamy EK. Diagnósticos de enfermagem de pacientes em tratamento radioterápico. Rev enferm UFPE on line., 2018; I2(I I):3060-8.
16. Okafor OO, Akinbami FO, Orimadegun AE, Okafor CM, Ogunbiyi AO. Prevalence of dermatological lesions in hospitalized children at the University College Hospital, Ibadan, Nigeria. Niger J Clin Pract. 201I; 14(3): 287-92.

17. Polat TAK, Yesilova Y, Alatas ET, Belli AA, Dogan G, Picakciefe M. Prevalence of skin diseases of the pediatric population in the Southeastern Anatolia. Medicine Science. 20I8; 7(3):664-7.

18. Santos CT, Almeida MA, Oliveira MC, Victor MAG, Lucena AF. Desenvolvimento do diagnóstico de enfermagem risco de úlcera por pressão. Rev Gaúcha Enferm. 20I5; 36(2): I I3-2I.

19. Oliveira IC, Veríssimo RCSS, Bastos MLA, Lúcio IML. A frequência dos diagnósticos de enfermagem em pacientes com feridas. Rev enferm UFPE on line, 20I4; 8(7):1937-46.
Recebido: 2019-10-06

Aceito: 2019-12-09 